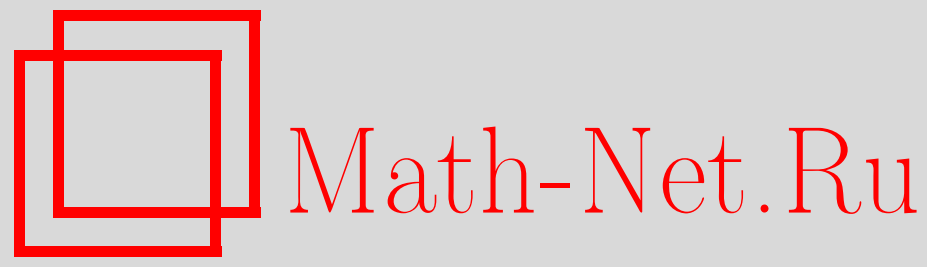

В. С. Буслаев, В. Ю. Страздин, Одномерный оператор Шрёдингера на полуоси: дифференциальное уравнение для собственных функций относительно спектрального параметра и аналог уравнения Фройда, Функи. анализ и его прил., 2007, том 41, выпуск 3, 84-88

DOI: https://doi.org/10.4213/faa2869

Использование Общероссийского математического портала MathNet.Ru подразумевает, что вы прочитали и согласны с пользовательским соглашением

http://www . mathnet.ru/rus/agreement

Параметры загрузки:

IP : 3.85 .183 .62

26 апреля 2023 г., 12:51:08

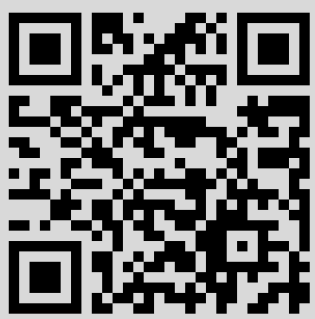




\section{Одномерный оператор Шрёдингера на полуоси: дифференциальное уравнение для собственных функций относительно спектрального параметра и аналог уравнения Фройда*}

(c) 2007. В. С. БуСлаев, В. Ю. СтраздиН

Об ортогональных многочленах. В спектральной теории матриц Якоби существуют два соотношения (см., например, [1], [2]), аналоги которых для оператора Шрёдингера, по-видимому, не известны. Кратко опишем эти соотношения. Удобно начать с рассмотрения системы собственных векторов, т. е. многочленов $P_{n}(\lambda)$, ортогональных на оси $\mathbb{R}$ относительно веса $w=\exp (-q)$, где $q=q(\lambda)$ - некоторая четная непрерывно дифференцируемая функция, которая при $|\lambda| \rightarrow \infty$ стремится к $+\infty$ степенным образом (последнее условие принято ради простоты изложения). Нормируем многочлены так, что $P_{n}(\lambda) \sim \lambda^{n}$ при $|\lambda| \rightarrow \infty$. Тогда

$$
\int_{\mathbb{R}} P_{n}(\lambda) P_{m}(\lambda) w(\lambda) d \lambda=h_{n} \delta_{n m},
$$

где $h_{n}$ - некоторые положительные коэффициенты. Перенормируем эти многочлены, положив $p_{n}(\lambda)=\left(h_{n}\right)^{-1 / 2} P_{n}(\lambda)$.

Соответствующая матрица Якоби $A$ действует на комплекснозначные последовательности $\mathbf{x}=\left\{x_{n}\right\}_{n=0}^{\infty}$ по правилу $(A \mathbf{x})_{n}=r_{n} x_{n-1}+r_{n+1} x_{n+1}, n>0$, и $(A \mathbf{x})_{0}=r_{1} x_{1}$. Здесь $r_{n}=\left(h_{n} / h_{n-1}\right)^{1 / 2}$. Векторы $\mathbf{p}(\lambda),(\mathbf{p}(\lambda))_{n}=p_{n}(\lambda)$, введенного выше пространства последовательностей образуют полную систему (обобщенных) собственных функций матрицы $A$, которая определяет самосопряженный оператор в $l_{2}(\mathbb{N}), \mathbb{N}=\{0, \ldots\}, A \mathbf{p}(\lambda)=\lambda \mathbf{p}(\lambda)$. Спектр матрицы $A$ однократен, непрерывен и совпадает с осью $\mathbb{R}$. При этом $\sum_{n \geqslant 0} p_{n}(\lambda) p_{n}(\mu) w(\mu)=$ $\delta(\lambda-\mu)$.

Первое из упомянутых соотношений имеет вид

$$
\begin{aligned}
& \frac{d}{d \lambda} p_{n}(\lambda)=r_{n} B_{n, n} p_{n-1}(\lambda)-r_{n} B_{n, n-1} p_{n}(\lambda), \\
& \text { где } B(\lambda)=\frac{W(A)}{A-\lambda-i 0}, W=-\frac{d}{d \lambda} \ln w(\lambda) .
\end{aligned}
$$

Второе соотношение - это классическое уравнение Фройда $r_{n}(W(A))_{n, n-1}=n$.

Некоторые факты спектральной теории оператора Шрёдингера. В настоящем разделе мы напоминаем свойства двух характерных решений уравнения Шрёдингера

$$
-y^{\prime \prime}+v(x) y=k^{2} y, \quad x>0,
$$

\footnotetext{
*Работа была поддержана грантами РФФИ 05-01-01076 и 05-01-02944.
} 
которые входят в ядро резольвенты $R(\lambda)=(L-\lambda I)^{-1}, \lambda=k^{2}$, оператора Шрёдингера $L \psi=-\psi^{\prime \prime}+v \psi, \psi(0)=0$. Можно считать, что потенциал $v(x)$, определяющий оператор и уравнение, веществен и суммируем на полуоси $(0, \infty)$ с весом $1+x^{2}$. Два характерных решения $\varphi(x, k)$ и $f(x, k)$ уравнения Шрёдингера определяются условиями $\varphi(0, k)=0, \varphi^{\prime}(0, k)=1, \lim _{x \rightarrow \infty} e^{-i k x} f(x, k)=1$; см. по поводу этих решений, например, [3].

Здесь и в дальнейшем мы будем использовать следующие обозначения для производных: $\varphi^{\prime}(x, k)=(\varphi(x, k))_{x}=\partial \varphi(x, k) / \partial x, \dot{\varphi}(x, k)=\partial \varphi(x, k) / \partial k$.

Перечислим некоторые свойства решений $\varphi(x, k)$ и $f(x, k)$, на которые мы будем ссылаться ниже.

Решение $\varphi(x, k)$ при каждом $x \geqslant 0$ является целой четной функцией параметра $k$, которая подчиняется оценкам

$$
\left|\varphi(x, k)-\frac{\sin k x}{k}\right| \leqslant K \frac{e^{|\operatorname{Im}(k)| x}}{1+|k|^{2}}, \quad\left|\frac{\partial}{\partial k}\left(\varphi(x, k)-\frac{\sin k x}{k}\right)\right| \leqslant K \frac{e^{|\operatorname{Im}(k)| x}}{1+|k|^{2}} .
$$

Решение $f(x, k)$ при каждом $x \geqslant 0$ аналитично по $k$ в верхней полуплоскости $\operatorname{Im}(k)>0$, непрерывно дифференцируемо по $k$ вплоть до вещественной оси и удовлетворяет оценкам

$$
\left|f(x, k) e^{-i k x}-1\right| \leqslant K \frac{1}{1+|k|}, \quad\left|\frac{\partial}{\partial k}\left(f(x, k) e^{-i k x}\right)\right| \leqslant K \frac{1}{1+|k|}, \quad \operatorname{Im}(k) \geqslant 0 .
$$

При вещественных $k$ выполняется соотношение $\varphi(x, k)=(2 i k)^{-1}(f(x, k) \overline{M(k)}$ $-\overline{f(x, k)} M(k))$, где $M(k)=f(0, k)$. Мы будем предполагать, что $M(0) \neq 0$, иначе говоря, $k=0$ не является виртуальным уровнем.

Спектр $\{\lambda\}$ оператора $L$ состоит из конечного числа отрицательных собственных значений и компоненты простого непрерывного спектра, заполняющей полуось $\lambda>0$. Чтобы изложение было короче, мы будем предполагать, что для рассматриваемого потенциала собственные значения отсутствуют. При сделанных предположениях функция $M(k)$ не имеет корней в замкнутой верхней полуплоскости. Функции $\varphi(x, k), \lambda=k^{2}$, являются обобщенными собственными функциями непрерывного спектра. Они удовлетворяют условиям ортонормированности и полноты:

$$
\begin{gathered}
\frac{2}{\pi} \int_{0}^{\infty} \varphi(y, k) \varphi(y, l) l \sigma(l) d y=\delta(k-l), \\
\frac{1}{\pi} \int_{0}^{\infty} \varphi(x, k) \varphi(y, k) \sigma(k) d \lambda=\delta(x-y), \quad \sigma(k)=\frac{k}{|M(k)|^{2}} .
\end{gathered}
$$

Как следствие, ядро $H(x, y)$ оператора $H=f(L)$ дается формулой

$$
H(x, y)=\frac{1}{\pi} \int_{0}^{\infty} f(\lambda) \varphi(x, k) \varphi(y, k) \sigma(k) d \lambda
$$


Спектральное разложение функции $\frac{1}{2 k} \dot{\varphi}(x, k)$. Умножим обе стороны соотношения ортонормированности на функцию $\frac{1}{2 l} \dot{\varphi}(x, l)$ и проинтегрируем полученное соотношение по $l$ по полуоси $l \geqslant 0$. Воспользуемся четностью подынтегральной функции относительно $l$ и распространим интеграл на всю ось:

$$
\frac{1}{2 k} \dot{\varphi}(x, k)=\frac{1}{\pi} \int_{0}^{\infty} C(x, y) \varphi(y, k) d y,
$$

где

$$
C(x, y)=\int_{0}^{\infty} \varphi(y, l) \dot{\varphi}(x, l) \frac{l}{|M(l)|^{2}} d l=\frac{1}{2} \int_{\mathbb{R}} \varphi(y, l) \dot{\varphi}(x, l) \frac{l}{|M(l)|^{2}} d l .
$$

Чтобы убедиться в том, что $C(x, y)$ является функцией точки (т. е. не имеет компонент, которые были бы сингулярными обобщенными функциями), подставим в последний интеграл вместо подынтегральной функции ее асимптотическое выражение при $l \rightarrow \infty$ :

$$
C(x, y) \sim \frac{1}{2} \int_{\mathbb{R}} \sin (l y) \frac{\partial}{\partial l}\left(\frac{\sin (l x)}{l}\right) d l .
$$

Сингулярная компонента последнего интеграла совпадает с сингулярной компонентой функции $C(x, y)$; нетрудно убедиться, что выписанный интеграл есть непрерывная функция переменных $x, y$.

Следующее наблюдение состоит в том, что $C(x, y)=0$ при $y>x$. Чтобы увидеть это, выразим функцию $\varphi(y, l)$ в интеграле, определяющем $C(x, y)$, через решение $f(y, l)$ :

$$
C(x, y)=\frac{1}{4 i} \int_{\mathbb{R}} \frac{f(y, l)}{M(l)} \dot{\varphi}(x, l) d l-\frac{1}{4 i} \int_{\mathbb{R}} \frac{f(y,-l)}{M(-l)} \dot{\varphi}(x, l) d l .
$$

Напомним, что функция $\varphi(y, l)$ - целая четная функция от $l$, а $f(y, l) / M(l)$ аналитична в верхней полуплоскости и непрерывна вплоть до вещественной оси, соответственно $f(y,-l) / M(-l)$ аналитична в нижней полуплоскости. Деформируя контуры интегрирования в выписанных интегралах в соответствующие полуплоскости, мы видим, с учетом асимптотических свойств подынтегральных функций, что при $y>x$ оба интеграла обращаются в нуль. Таким образом,

$$
\frac{1}{2 k} \dot{\varphi}(x, k)=\frac{1}{\pi} \int_{0}^{x} C(x, y) \varphi(y, k) d y, \quad C(x, y)=\frac{1}{2} \int_{\mathbb{R}} \varphi(y, l) \dot{\varphi}(x, l) \frac{l}{|M(l)|^{2}} d l .
$$

При $y<x$ функция $C(x, y)$ допускает дальнейшие преобразования. Выполним в последнем интеграле интегрирование по частям, перенося производную с $\dot{\varphi}(x, l)$ на два других множителя:

$C(x, y)=-\frac{1}{2} \int_{\mathbb{R}} \dot{\varphi}(y, l) \varphi(x, l) \frac{l}{|M(l)|^{2}} d l-\frac{1}{2} \int_{\mathbb{R}} \varphi(y, l) \varphi(x, l) \tau(l) d l, \quad \tau(l)=\frac{d}{d l} \sigma(l)$.

В силу доказанного выше первый интеграл в правой части при $y<x$ равен нулю; поэтому

$$
C(x, y)=-\frac{1}{2} \int_{\mathbb{R}} \varphi(y, l) \varphi(x, l) \tau(l) d l=-\int_{0}^{\infty} \varphi(y, l) \varphi(x, l) \tau(l) d l .
$$


Дифференциальное уравнение относительно переменной $\lambda$. Вернемся к спектральному представлению функции $\frac{1}{2 k} \dot{\varphi}(x, k)$ :

$$
\frac{1}{2 k} \dot{\varphi}(x, k)=-\frac{1}{\pi} \int_{0}^{\infty} \varphi(x, l) \tau(l) d l \int_{0}^{x} \varphi(y, k) \varphi(y, l) d y .
$$

Из дифференциального уравнения для $\varphi(x, k)$ относительно $x$ стандартным образом выводится соотношение

$$
\int_{0}^{x} \varphi(y, k) \varphi(y, l) d y=\frac{\varphi(x, k) \varphi^{\prime}(x, l)-\varphi(x, l) \varphi^{\prime}(x, k)}{k^{2}-l^{2}} .
$$

Таким образом,

$$
\frac{1}{2 k} \dot{\varphi}(x, k)=\frac{1}{\pi} \int_{0}^{\infty} \frac{\varphi(x, l) \varphi(x, l) \varphi^{\prime}(x, k)-\varphi(x, l) \varphi^{\prime}(x, l) \varphi(x, k)}{k^{2}-l^{2}} \tau(l) d l .
$$

Два появившихся здесь интеграла можно расчленить, если подходящим и одинаковым образом интерпретировать в обоих слагаемых, которые должны при этом возникнуть, сингулярный знаменатель $\left(k^{2}-l^{2}\right)^{-1}$. Как он будет интерпретирован - неважно. Важно, что он должен быть интерпретирован как одна и та же обобщенная функция в обоих слагаемых. Это может быть главное значение или предельное значение с определенной сторонъ, или что-либо другое. Просто для того, чтобы подчеркнуть, что какая-то регуляризация необходима, мы будем в дальнейшем использовать конкретную регуляризацию $\left(k^{2}-l^{2}\right)^{-1}=$ $\left(k^{2}-l^{2}+i 0\right)^{-1}$.

С этой договоренностью

$$
\frac{\partial}{\partial \lambda} \varphi(x, k)=M(x, x, \lambda) \varphi^{\prime}(x, k)-\left.M_{y}(x, y, \lambda)\right|_{y=x} \varphi(x, k),
$$

где

$$
\begin{gathered}
M(x, y, \lambda)=\frac{1}{\pi} \int_{0}^{\infty} \frac{\varphi(x, l) \varphi(y, l)}{k^{2}-l^{2}+i 0} \tau(l) d l=\frac{1}{\pi} \int_{0}^{\infty} \frac{\varphi(x, l) \varphi(y, l)}{\mu-\lambda-i 0} \sigma(l) W(\mu) d \mu, \\
W(\mu)=-\frac{d}{d \mu} \ln \sigma(l), \quad \mu=l^{2} .
\end{gathered}
$$

Коэффициенты уравнения можно переписать несколько иначе:

$$
\frac{\partial}{\partial \lambda} \varphi(x, k)=N(x, \lambda) \varphi^{\prime}(x, k)-\frac{1}{2} N_{x}(x, \lambda) \varphi(x, k), \quad N(x, \lambda)=M(x, x, \lambda) .
$$

Ясно, что $M(x, y, \lambda)$ можно записать как ядро оператора $M, M=W(L)(L-$ $\lambda-i 0)^{-1}$.

Чтобы получить замкнутую систему для вектора $\Phi(x, \lambda)=\left(\varphi(x, k), \varphi^{\prime}(x, k)\right)^{t}$, продифференцируем уравнение $(20)$ по $x$ и исключим из полученного соотношения производную $\varphi^{\prime \prime}(x, k)$, используя уравнение Шрёдингера. В результате приходим к следующей теореме:

Теорема 1. При сделанных выше предположениях относительно потенииала $v$ и спектральных свойств оператора $L$ вектор-функиия $\Phi(x, \lambda)=(\varphi(x, k)$, 
$\left.\varphi^{\prime}(x, k)\right)^{t}$ удовлетворяет уравнению

$$
\begin{gathered}
\frac{\partial}{\partial \lambda} \Phi(x, \lambda)=U(x, \lambda) \Phi(x, \lambda), \\
U(x, \lambda)=\left(\begin{array}{cc}
-\frac{1}{2} N_{x}(x, \lambda) & N(x, \lambda) \\
N(x, \lambda)(v(x)-\lambda)-\frac{1}{2} N_{x x}(x, \lambda) & \frac{1}{2} N_{x}(x, \lambda)
\end{array}\right) .
\end{gathered}
$$

Выполняется также соотношение $-2(W(L)(x, x))_{x}=1$. Определения функиии $N$ и оператора $W$ даны выше.

Для доказательства последнего утверждения теоремы необходимы дополнительные вычисления. Обратимся к ним.

Струнное уравнение. В виде системы, аналогичной предыдущему уравнению, можно записать и само уравнение Шрёдингера:

$$
\frac{\partial}{\partial x} \Phi(x, \lambda)=V(x, \lambda) \Phi(x, \lambda), \quad \text { где } V(x, \lambda)=\left(\begin{array}{cc}
0 & 1 \\
v(x)-\lambda & 0
\end{array}\right) .
$$

Условие совместности двух последних уравнений имеет вид $\partial U / \partial x=\partial V / \partial \lambda+$ $[V, U]$. Только элемент $(2,1)$ этого условия ведет к содержательному соотношению: $\frac{1}{2} N_{x x x}-2(v(x)-\lambda) N_{x}-v^{\prime}(x) N=1$.

Заметим, что соответствующему однородному уравнению третьего порядка удовлетворяет произведение двух произвольных решений уравнения Шрёдингера. Отсюда немедленно следует, что

$$
\begin{aligned}
\frac{1}{2} N_{x x x}-2(v(x)-\lambda) N_{x}-v^{\prime}(x) N & =\frac{1}{\pi} \int_{0}^{\infty} 2(\lambda-\mu) \frac{(\varphi(x, l) \varphi(x, l))_{x}}{\mu-\lambda-i 0} \sigma(l) W(\mu) d \mu \\
& =-\frac{2}{\pi} \int_{0}^{\infty}(\varphi(x, l) \varphi(x, l))_{x} \sigma(l) W(\mu) d \mu,
\end{aligned}
$$

и, таким образом, $-2(W(L)(x, x))_{x}=1$.

Это и есть аналог уравнения Фройда для ортогональных многочленов. Есть много оснований считать, что это уравнение связано с иерархией Пенлеве-I интегрируемых обыкновенных дифференциальных уравнений, возникшей в теории двумерной квантовой гравитации (см., например, [4]).

Первый автор благодарит А. Р. Итса за полезные обсуждения. Он указал нам, в частности, на возможную связь аналога уравнения Фройда с уравнениями иерархии Пенлеве-I.

\section{ЛитеРАТУРА}

[1] A. S. Fokas, A. R. Its, A. V. Kitaev, Comm. Math. Phys., 147:2 (1992), 395-430. [2] Yang Chen, Mourad E. H. Ismail, J. Phys. A: Math. Gen., 30 (1997), 7817-7829. [3] Л. Д. Фаддеев, УМH, 14:4 (1959), 57-119. [4] G. Moore, Comm. Math. Phys., 133:2 (1990), 261-304.

Санкт-Петербургский государственный университет e-mail: buslaev@mph.phys.spbu.ru

Поступило в редакцию Санкт-Петербургский государственный университет e-mail: strazdin81@mail.ru 